\title{
ACTINOMYCOSIS PRESENTING AS SUPERIOR VENA CAVA SYNDROME IN A YOUNG PUERPERAL WOMAN
}

\author{
Antonios Loutsidis, MD, ${ }^{\mathrm{a}}$ Charalambos Zisis, $\mathrm{MD},{ }^{\mathrm{a}}$ Dimitra Rontogianni, $\mathrm{MD},{ }^{\mathrm{b}}$ and Ion Bellenis, MD, FRCS, ${ }^{\mathrm{a}}$ Athens, Greece
}

Thoracic actinomycosis is a rare pathologic condition frequently masquerading as cancer of the lung. The mistaken diagnosis may result in thoracotomy and pulmonary resection as treatment, given that the majority of the cases initially remain undiagnosed. Because of the high mortality of the disease, if untreated, it is imperative to obtain the diagnosis as soon as possible. Appropriate treatment may ensure a good prognosis.

From the Department of Thoracic and Vascular Surgery a and the Department of Pathology, ${ }^{\mathrm{b}}$ Evangelismos General Hospital, Athens, Greece.

Received for publication April 10, 2000; accepted for publication May 5, 2000.

Address for reprints: Antonios Loutsidis, Omirou 19, 16675 Glyfada, Athens, Greece (E-mail: dkakats@cc.uoa.gr).

J Thorac Cardiovasc Surg 2000;120:1009-10

Copyright (C) 2000 by The American Association for Thoracic Surgery

$0022-5223 / 2000 \$ 12.00+0 \quad \mathbf{1 2 / 5 4 / 1 0 8 5 2 8}$

doi: $10.1067 / \mathrm{mtc} .2000 .108528$
Clinical summary. A 19-year-old woman from Albania was admitted for respiratory distress. One month previously, she had a normal uncomplicated delivery. Fifteen days later, leg edema, left-sided chest pain, and progressive dyspnea developed. On admission, physical examination revealed an ill-looking woman with a respiratory rate of 40 breaths/min, a pulse rate of 150 beats/min, an arterial blood pressure of $80 / 40 \mathrm{~mm} \mathrm{Hg}$, and a temperature of $38.3^{\circ} \mathrm{C}$. Ascites and pleural fluid were detected on percussion and auscultation, and veins in the neck were dilated. Computed tomographic (CT) scan of the thorax demonstrated an extended mass of the anterior and middle mediastinum, exerting pressure on the right subclavian vein and superior vena cava. An extrinsic compression on the right superior lobar bronchus, atelectasis of the middle lobe, and right pleural effusion were revealed (Fig 1). CT scanning of the abdomen showed extensive hepatomegaly and prominent abdominal effusion. Echocardiography revealed an extracardiac mass encircling the posterior surface of the right and left atria and partly encircling the right and left ventricles. This mass resulted in compression of the superior vena cava and distention of the inferior vena cava. On diagnostic aspiration, the pleural fluid 


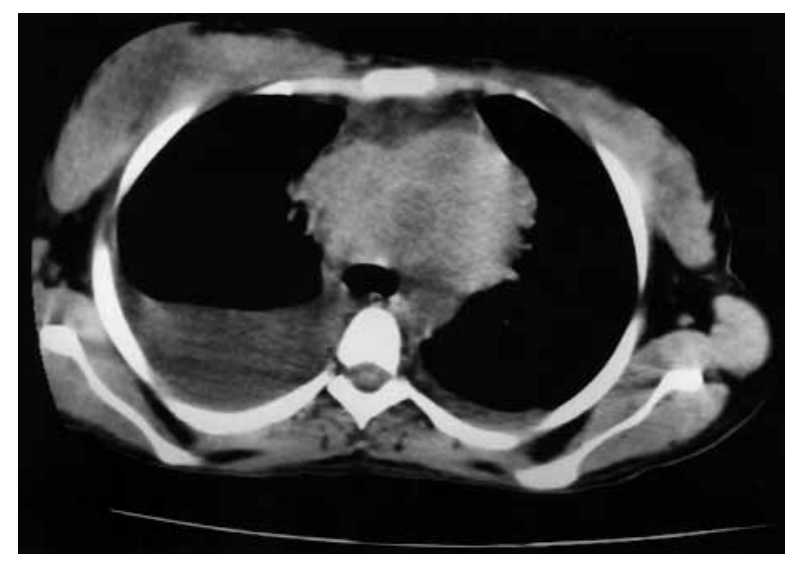

Fig 1. The mass of the anterosuperior mediastinum in contact with superior vena cava and concomitant right pleural effusion.

proved to be an exudate with $65 \%$ lymphocytes, $35 \%$ neutrophils, and some mesothelial cells, whereas Gram and ZielNielsen stains were negative. Many macrophages and mesothelial cells were also detected in the ascitic fluid. Primary lymphoma of the mediastinum was considered to be the most probable pathologic entity. Because mediastinoscopy was considered risky, a mediastinotomy was preferred to obtain sufficient tissue for accurate histopathologic diagnosis. Even though this technique was performed, excessive bleeding occurred as a result of injury to the dilated veins of the superior mediastinum. Multiple biopsy specimens were needed from the mass to establish a diagnosis because all frozen sections were negative for malignant disease. Histologic examination of permanent sections showed actinomycosis of the mediastinum. The characteristic organisms were identified in the inflammatory material as clusters surrounded by sulfur granules. The methenamine silver procedure was used for staining the filaments (Fig 2). The patient was managed with intravenous administration of 30,000,000 IU of penicillin per day. She responded well had had a speedy recovery.

Discussion. Thoracic actinomycosis is frequently confused with lung cancer, lymph node blocks, or mediastinal tumors. One case of thoracic actinomycosis with superior vena cava obstruction has been described in the literature, with lethal cerebral hemorrhage before the intended operation. ${ }^{1}$ The thoracic variety of the disease accounts for approximately $15 \%$ of the cases..$^{2,3}$ Thoracic pain is considered the first symptom in many cases, ${ }^{2}$ but the examination and inspection of available material stained by direct microscopy is crucial. ${ }^{4}$ Thoracic actinomycosis is attributed to the aspiration of oropharyngeal secretions. ${ }^{3,4}$ The usual mechanism of the thoracic variety is by infection of the lung. In the presented case, atelectasis of the middle lobe and pleural effusion suggest pulmonary infection. This
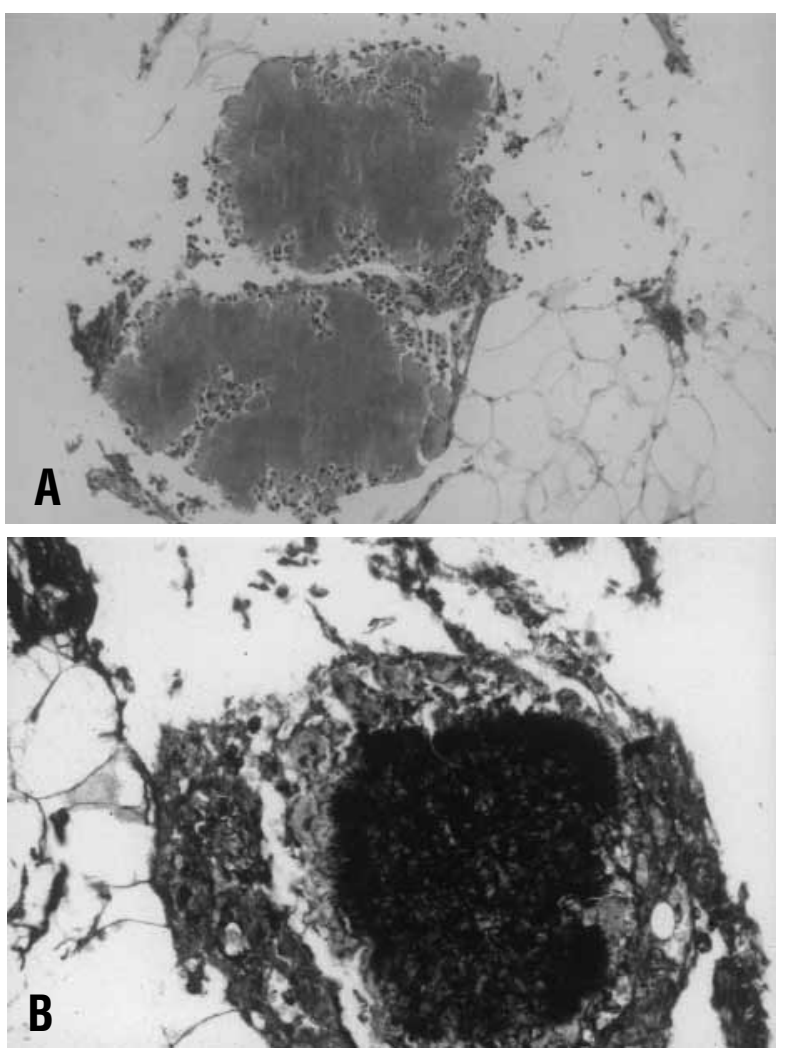

Fig 2. A, Colonies of the actinomyces in the purulent material. (Hematoxylin and eosin stain; original magnification $\times 100$.) B, Filaments of the actinomyces stained by methenamine silver procedure. (Methenamine silver stain; original magnification $\times 200$.)

infection is considered to extend across fissures and through the pleura. ${ }^{3}$ Mediastinal actinomycosis is provoked by direct local extension from the neck. Air space consolidation with adjacent pleural thickening are radiologic findings suggestive of pulmonary actinomycosis. ${ }^{3} \mathrm{CT}$ findings overlap those of other inflammatory and neoplastic conditions, including soft tissue mass, with various degrees of infiltration and abscess formation. ${ }^{5}$

\section{REFERENCES}

1. Schmidt G, Fuessl H. Thoracic actinomycosis with superior vena cava obstruction. Deutsch Med Wochenschr 1979;104:1607-9.

2. Moller-Jensen B, Kruse-Andersen S, Andersen K. Thoracopleural actinomycosis presenting like diffuse pulmonary embolism. Thorac Cardiovasc Surg 1988;36:284-6.

3. Poey C, Giron J, Verhaegen F, Levenes H, Gruels S, Fajadet P, et al. X-ray computed tomography and radiographic aspects of thoracic actinomycosis. J Radiol 1996;77:177-83.

4. Bentley ER, Ostranscy D. Unusual manifestations of thoracic actinomycosis. J Am Osteopath Assoc 1994;94:249-53.

5. Allen HA 3rd, Scatarige JC, Kim MH. Actinomycosis: CT findings in six patients. AJR Am J Roentgenol 1987;149:1255-8. 